\title{
Follow-Up Assessment of Health Consequences after a Chlorine Release from a Train Derailment-Graniteville, SC, 2005
}

\author{
Mary Anne Duncan • Daniel Drociuk • Amy Belflower-Thomas • David Van Sickle • \\ James J. Gibson • Claire Youngblood • W. Randolph Daley
}

Published online: 2 February 2011

(C) American College of Medical Toxicology 2011

\begin{abstract}
Introduction After a train derailment released chlorine gas in Graniteville, South Carolina, in 2005, a multiagency team performed an epidemiologic assessment of chlorine exposure and resulting health effects. Five months later, participants were resurveyed to determine their health status and needs and to assist in planning additional interventions in the community.

Methods Questionnaires were mailed to 279 patients interviewed in the initial assessment; follow-up telephone calls were made to nonresponders. The questionnaire included questions regarding duration of symptoms experienced after exposure and a posttraumatic stress disorder (PTSD) assessment tool.

Results Ninety-four questionnaires were returned. Seventysix persons reported chronic symptoms related to the
\end{abstract}

M. A. Duncan · D. Van Sickle

Epidemic Intelligence Service, Centers for Disease Control and Prevention,

Atlanta, GA, USA

M. A. Duncan · D. Drociuk · A. Belflower-Thomas •

C. Youngblood

Division of Acute Disease Epidemiology, South Carolina

Department of Health and Environmental Control,

Columbia, SC, USA

D. Van Sickle

Air Pollution and Respiratory Health Branch,

National Center for Environmental Health,

Centers for Disease Control and Prevention,

Atlanta, GA, USA

J. J. Gibson

Bureau of Disease Control, South Carolina Department of Health and Environmental Control,

Columbia, SC, USA chlorine exposure, 47 were still under a doctor's care, and 49 were still taking medication for chlorine-related problems. Agreement was poor between the first and second questionnaires regarding symptoms experienced after exposure to the chlorine $(\kappa=0.30)$. Forty-four respondents screened positive for PTSD. PTSD was associated with post-exposure hospitalization for three or more nights [relative risk $(\mathrm{RR})=1.7 ; 95 \%$ confidence interval $(\mathrm{CI})=$ 1.1-2.6] and chronic symptoms $(\mathrm{RR}=9.1 ; 95 \% \mathrm{CI}=1.3$ $61.2)$, but not with a moderate-to-extreme level of chlorine exposure $(\mathrm{RR}=1.2 ; 95 \% \mathrm{CI}=0.8-1.8)$.

Conclusions Some victims of this chlorine exposure event continued to experience physical symptoms and continued to require medical care 5 months later. Chronic mental health symptoms were prevalent, especially among persons experiencing the most severe or persistent physical health

W. R. Daley

Epidemic Intelligence Service Field Assignments Branch,

Centers for Disease Control and Prevention,

Atlanta, GA, USA

Present Address:

M. A. Duncan $(\bowtie)$

Division of Health Studies, Agency for Toxic Substances

and Disease Registry,

4770 Buford Highway NE, MS F-57,

Chamblee, GA 30341, USA

e-mail: maduncan@cdc.gov

Present Address:

A. Belflower-Thomas

Health Education Division, Pitt County Health Department,

Greenville, NC, USA

Present Address:

D. Van Sickle

Reciprocal Labs Corporation,

Madison, WI, USA 
effects. Patients should be interviewed as soon as possible after an incident because recall of acute symptoms experienced can diminish within months.

Keywords Chlorine $\cdot$ Chemical release $\cdot$ Train derailment

\section{Introduction}

At approximately 2:40 A.M. on 6 January 2005, a train derailment caused the release of approximately 60 tons of chlorine gas in the center of Graniteville, South Carolina. The incident occurred on the grounds of a textile mill where approximately 180 persons were working the night shift.

In response to the chlorine spill, the Division of Acute Disease Epidemiology and the regional and county offices of the South Carolina Department of Health and Environmental Control (DHEC), in collaboration with the Centers for Disease Control and Prevention, began a rapid epidemiologic assessment. The objectives of this assessment were as follows: to determine the extent and location of exposure, assess morbidity caused by chlorine, examine health services use related to the incident, identify risk factors for more severe outcomes, and determine which persons were at risk for long-term sequelae. A questionnaire was developed, and patients were interviewed either in person, at hospitals, or by telephone.

A case was defined as death or illness attributed to chlorine exposure in a patient treated at a local health care facility, reported 6 January-17 February 2005, from the Graniteville area. A total of 605 cases were identified. Nine deaths occurred as a result of the chlorine release, eight at the scene and one at a hospital. A total of 597 persons sought medical care after chlorine exposure; 72 (12\%) were hospitalized and $525(88 \%)$ were examined as outpatients in hospital emergency departments or at private physicians' offices. Interviews were completed with 280 patients or their proxies. The findings of the investigation have been published in a prior report [1].

Five months after the derailment, participants of the rapid assessment were resurveyed to determine their health status and needs and to assist in planning additional interventions in the community. The objectives of the follow-up assessment were to determine the current health status of patients, learn the duration of symptoms experienced related to the chlorine exposure, and determine the proportion of respondents that had symptoms suggestive of posttraumatic stress disorder (PTSD). In addition, assistance was provided to those requesting help with obtaining referrals for medical care, counseling, or financial aid. This report describes the findings of the follow-up assessment of the health consequences of the chlorine release.

\section{Methods}

Exposure and Outcome Measures Used in the Rapid Assessment

During the rapid assessment, patients were assigned severity-of-exposure ratings by a panel of team members on the basis of patient location during exposure, duration of exposure, description of exposure, and any symptoms described to an interviewer; the ratings included no exposure, mild, moderate, high, and extreme exposures. During the analysis, the categories were dichotomized by combining the no-to-mild exposure ratings and moderateto-extreme exposure ratings. A complete description of the process of assigning the ratings has been reported elsewhere [2].

Medical care required after the chlorine exposure was used as the outcome measure in the rapid assessment. Patients who were hospitalized three or more nights and those who died as a result of the chlorine were classified as having a severe medical outcome. Patients hospitalized for one or two nights and those receiving care as an outpatient were classified as having a less severe medical outcome.

\section{Questionnaire}

A questionnaire was developed for the follow-up assessment. Questions included whether the person was still experiencing symptoms that he or she believed were related to the chlorine exposure and whether or not the person was still under a doctor's care or taking medicine for problems related to the chlorine. Respondents were asked if they had experienced any of a list of symptoms after exposure to the chlorine; the list included the symptoms asked about during the rapid assessment, additional commonly reported symptoms (e.g., throat burning or nose bleeds), and the longer term problem of memory loss. Ringing of the ears, a symptom not known to be associated with chlorine exposure and not reported by any participants during the rapid assessment, was also included to help determine if patients would report experiencing any symptom about which we asked. Respondents were also asked which symptoms were still present. Patients were asked to check a box that best described the duration of any symptoms that had resolved at the time of the follow-up assessment.

The 17-question Posttraumatic Stress Disorder Checklist for a Specific Event (PCL-S) [3, 4] was included in the questionnaire. This instrument includes questions on how often a series of problems had bothered the respondent within the previous month.

Respondents were asked whether they had attended any community meetings about the train wreck and, if so, whether the meeting had been helpful in keeping the person 
informed or whether it had provided emotional support. They were also asked whether they needed assistance obtaining medical care, supportive counseling, or information.

In the cover letter, parents were asked to answer for their children. The questionnaires were mailed to the addresses given during the rapid assessment interviews. Those returned as undeliverable were remailed if a correct address was located. Telephone calls were made to nonresponders and those whose correct address could not be determined. Those who were reached were offered the option of completing the questionnaire by telephone interview. If they preferred to answer by mail, a second copy of the questionnaire was mailed. No further attempts were made to reach nonresponders because the DHEC Community Health Survey, which included free physical exams to persons exposed to the chlorine, was beginning and we did not want to have multiple DHEC assessments occurring simultaneously.

\section{Data Management and Analysis}

Data were entered into a Microsoft $^{\circledR}$ Access $^{\circledR}$ database; double data entry was employed to ensure accuracy. Analysis was performed using SAS ${ }^{\circledR} 9.1$ [5] and included descriptive analysis, contingency table analysis, analysis of variance, and calculation of the kappa coefficient comparing symptoms reported on the rapid assessment with those reported on the follow-up assessment. A comparison of proportions and means was performed with Epi Info $^{\mathrm{TM}}$ 6.04d [6]. Not all participants answered every question on the questionnaire; observations with missing data for a variable were only excluded from the analysis of that variable.

Methodology for evaluating the PCL-S includes either using the raw score or having symptomatic responses (scores of 3-5) from at least one of questions 1-5, three of questions 6-12, and two of questions 13-17. In this assessment, we considered a person as having screened positive for PTSD if he or she had a raw score of $\geq 44$ and symptomatic responses to at least the minimum number in each of three groups of questions.

\section{Results}

Questionnaires were mailed to 279 persons who had been interviewed during the rapid assessment; 94 (34\%) questionnaires were returned.

\section{Demographic Characteristics}

Demographic characteristics, exposure severity rating, and outcome classification of respondents are displayed in
Table 1; the characteristics of the persons who had sought medical care for chlorine exposure after the derailment and who had been identified during the rapid assessment are included for comparison. The mean age of respondents during the follow-up assessment was 41 years (range, 281 years). Sixty-four percent of respondents were male; $69 \%$ were white and $29 \%$ were black. These demographic characteristics are not statistically different from the total group identified who had sought medical care. In the follow-up assessment, a higher proportion of persons had been exposed to the chlorine at their Graniteville residences (40\% versus $33 \%$ ), and a lower proportion had been exposed while working at the mill (26\% versus $31 \%)$, but these proportions were not statistically different.

The proportion of persons experiencing a moderate, high, or extreme chlorine exposure, compared with an unidentifiable or low exposure, was likewise similar. Respondents to the follow-up assessment were more likely to have experienced a severe medical outcome than the total group of persons identified during the rapid assessment who had sought medical care after chlorine exposure $(16 \%$ versus $8 \%, p=0.02$ ).

\section{Results of Questionnaire}

The respondents' health status, the proportion still under a doctor's care and still taking medicine, and their ability to obtain care are displayed in Table 2. Also listed in Table 2 is the risk for having an affirmative answer to each question for those who had experienced a severe medical outcome compared with those who had experienced a less severe outcome. Of the 92 responses received, $83 \%$ reported that they were still experiencing symptoms that they believed were related to chlorine exposure from the derailment, and approximately half reported being under a physician's care and taking medicine for these problems. Persons who had experienced a severe medical outcome were significantly more likely to still be experiencing symptoms, be under a physician's care, and be taking medication than were persons who had a less severe medical outcome.

Respondents reported a median of one emergency department visit (range, 0-6) and a median of three visits to doctors' offices (range, 0-22). Twenty-nine of 86 (34\%) patients responding to this question reported that they had attended one or more community meetings (median, 2; range, 1-3); of 25 attendees responding, 14 (56\%) reported that these events were helpful in keeping them informed or providing emotional support.

Table 3 indicates the acute symptoms that respondents experienced after chlorine exposure. As in the rapid assessment, coughing and eye burning were the most commonly reported symptoms. Table 3 also indicates the number of persons for whom the symptom resolved during 
Table 1 Demographic information of participants in follow-up assessment compared with those who sought medical care for chlorine exposure identified during the rapid assessment after the train derailment
Demographic information

Follow-up assessment $(n=94)$ $n(\%)$

Rapid assessment $(n=605)^{\mathrm{a}}$ $n(\%)$
${ }^{a}$ Because of missing data, percentages are based on the following sample sizes: sex, 565; race/ethnicity, 426, patient category, 331; exposure severity rating, 292

${ }^{\mathrm{b}}$ Other responses for race/ethnicity included Native American, Pacific Islander, Indian, and multiracial or ethnic backgrounds

${ }^{\mathrm{c}}$ Patient category describes where the person was at the time of exposure. Night-shift mill workers were categorized as "worker at mill," even if they lived in Graniteville. Day-shift mill workers who lived in Graniteville were categorized as "Graniteville residents"

\begin{tabular}{|c|c|c|}
\hline \multicolumn{3}{|l|}{ Age (in years) } \\
\hline Mean & 41 & 36 \\
\hline Range & $2-81$ & $<1-85$ \\
\hline \multicolumn{3}{|l|}{ Sex } \\
\hline Male & $60(63.8)$ & $332(58.8)$ \\
\hline Female & $34(36.2)$ & $233(41.2)$ \\
\hline \multicolumn{3}{|l|}{ Race/ethnicity } \\
\hline White & $65(69.2)$ & $255(59.9)$ \\
\hline Black & $27(28.7)$ & $150(35.2)$ \\
\hline Hispanic & $1(1.1)$ & $11(2.6)$ \\
\hline Other $^{b}$ & $1(1.1)$ & $10(2.4)$ \\
\hline \multicolumn{3}{|l|}{ Patient category ${ }^{\mathrm{c}}$} \\
\hline Graniteville resident & $38(40.4)$ & $110(33.2)$ \\
\hline Worker at mill & $24(25.5)$ & $103(31.1)$ \\
\hline Resident of contiguous town & $10(10.6)$ & $23(7.0)$ \\
\hline In vehicle close to site & $8(8.5)$ & $36(10.9)$ \\
\hline Resident of noncontiguous town & $4(4.3)$ & $19(5.7)$ \\
\hline Rescue worker & $4(4.3)$ & $12(3.6)$ \\
\hline In vehicle farther from site & $3(3.2)$ & $21(6.3)$ \\
\hline Other & $3(3.2)$ & $7(2.1)$ \\
\hline \multicolumn{3}{|l|}{ Exposure severity rating } \\
\hline Moderate-to-extreme exposure & $45(47.9)$ & $145(49.7)$ \\
\hline No or low exposure & $49(52.1)$ & $147(50.3)$ \\
\hline \multicolumn{3}{|l|}{ Medical outcome } \\
\hline Severe medical outcome & $15(16.0)$ & $51(8.4)$ \\
\hline Less severe medical outcome & $79(84.0)$ & $544(91.6)$ \\
\hline
\end{tabular}

the period after the derailment. This table also displays the number of persons still experiencing the symptom at the time of the resurvey, 5 months after the derailment. The persistent symptoms reported most commonly were memory loss and shortness of breath; 27 of 38 (71\%) respondents who reported memory loss and 49 of 70 $(70 \%)$ who reported shortness of breath after the derailment continued to experience these symptoms at the time of the follow-up assessment.
Agreement between Acute Symptoms Reported on the Rapid and Follow-up Assessments

To assess the accuracy of recalled self-reported symptoms 5 months after a chemical event, we compared the answer each participant gave when asked if he or she had experienced a symptom after exposure to chlorine on the follow-up assessment with the answer given on the rapid assessment.
Table 2 Health status of respondents in the follow-up assessment 5 months after the chlorine release

\footnotetext{
${ }^{a}$ Because of missing responses, $n$ varies from 70 to 94

${ }^{\mathrm{b}} \mathrm{RR}$ and $95 \% \mathrm{CI}$ of an affirmative answer for patients classified with a severe medical outcome compared with those who had a less severe medical outcome
}

\begin{tabular}{llll}
\hline Current status $^{\mathrm{a}}$ & $n(\%)$ & $\mathrm{RR}^{\mathrm{b}}$ & $95 \% \mathrm{CI}^{\mathrm{b}}$ \\
\hline Still experiencing symptoms & $76(82.6)$ & 1.3 & $1.1-1.4$ \\
Still under doctor's care & $47(51.1)$ & 1.8 & $1.2-2.5$ \\
Still taking medicine & $49(52.1)$ & 1.9 & $1.4-2.6$ \\
Able to obtain needed medical care & $70(78.7)$ & 1.1 & $0.9-1.4$ \\
Able to obtain needed medicine & $62(74.7)$ & 1.0 & $0.8-1.4$ \\
Effects of chlorine kept/keeping from work & $49(59.0)$ & 1.8 & $1.4-2.3$ \\
Trouble breathing limits walking & $50(58.8)$ & 1.7 & $1.3-2.3$ \\
Most recent doctor visit was after June 1, 2005 & $20(28.6)$ & 2.0 & $0.9-4.3$ \\
\hline
\end{tabular}


Table 3 Symptoms experienced after exposure to chlorine that were reported during the follow-up assessment and symptom duration

\begin{tabular}{|c|c|c|c|c|c|c|c|c|}
\hline \multirow[t]{2}{*}{ Symptom } & \multirow{2}{*}{$\begin{array}{l}\text { Experienced } \\
\text { symptom } \\
n^{\mathrm{a}}(\%)\end{array}$} & \multicolumn{6}{|c|}{ No. of persons with symptom resolving within each period ${ }^{\mathrm{b}}$} & \multirow{2}{*}{$\begin{array}{l}\text { Symptom } \\
\text { persistent } \\
n(\%)^{\mathrm{d}}\end{array}$} \\
\hline & & $<1$ week & $1-2$ weeks & $2-4$ weeks & $1-2$ months & $2-3$ months & $>3$ months & \\
\hline Coughing & $80(93.0)$ & 4 & 7 & 7 & 9 & 1 & 1 & $44(55.0)$ \\
\hline Eye burning & $70(82.4)$ & 14 & 11 & 5 & 3 & 2 & 1 & $25(35.7)$ \\
\hline Shortness of breath & $70(81.4)$ & 4 & 2 & 2 & 2 & 3 & 2 & $49(70.0)$ \\
\hline Headache & $68(80.0)$ & 9 & 3 & 4 & 2 & 4 & 2 & $36(52.9)$ \\
\hline Coughing up phlegm/mucus & $60(75.0)$ & 1 & 6 & 7 & 2 & 2 & 2 & $32(53.3)$ \\
\hline Throat burning & $61(73.5)$ & 9 & 8 & 3 & 5 & 0 & 1 & $26(42.6)$ \\
\hline Nausea & $59(72.0)$ & 9 & 6 & 6 & 2 & 3 & 1 & $24(40.7)$ \\
\hline Nose burning & $54(70.1)$ & 15 & 6 & 3 & 3 & 1 & 1 & $18(33.3)$ \\
\hline Chest pain/burning & $54(69.2)$ & 5 & 6 & 2 & 1 & 1 & 1 & $29(53.7)$ \\
\hline Dizziness & $54(69.2)$ & 6 & 4 & 4 & 3 & 3 & 2 & $26(48.1)$ \\
\hline Blurred vision & $49(63.6)$ & 3 & 4 & 3 & 0 & 0 & 1 & $26(53.1)$ \\
\hline Choking & $43(58.9)$ & 6 & 7 & 0 & 4 & 1 & 0 & $18(41.9)$ \\
\hline Skin irritation/rash & $37(52.1)$ & 0 & 8 & 5 & 2 & 0 & 1 & $17(45.9)$ \\
\hline Memory loss & $38(48.7)$ & 1 & 0 & 2 & 0 & 1 & 0 & $27(71.1)$ \\
\hline Vomiting & $30(41.1)$ & 9 & 4 & 4 & 0 & 0 & 0 & $9(30.0)$ \\
\hline Ringing of the ears & $27(34.6)$ & 4 & 3 & 2 & 0 & 2 & 1 & $11(40.7)$ \\
\hline Nose bleeds & $25(32.9)$ & 4 & 1 & 0 & 1 & 1 & 2 & $12(48.0)$ \\
\hline
\end{tabular}

${ }^{\text {a }}$ Because of missing responses, $n$ varies from 71 to 86

${ }^{\mathrm{b}}$ Not all patients who reported that a symptom resolved listed its duration

${ }^{\mathrm{c}}$ Symptom still being experienced at time of follow-up assessment, 5 months after the derailment

${ }^{d}$ Percentage of persons who reported experiencing the symptom after the derailment who were still experiencing it at the time of the follow-up assessment

A kappa coefficient was calculated to evaluate the degree of agreement beyond chance between report of acute symptoms each person experienced on the rapid assessment and the follow-up assessment (Table 4). A kappa of $\geq 0.75$ indicates excellent agreement, $0.40-0.75$ indicates moderate agreement, and $<0.40$ is indicative of poor agreement [7]. Only chest pain $(\kappa=0.56)$, shortness of breath $(\kappa=0.49)$, and eye burning $(\kappa=0.44)$ were in the moderate agreement range; kappa scores for the other symptoms indicate poor agreement between recalled symptoms across assessments. The weighted average kappa coefficient for all symptoms was 0.30 .

On the follow-up assessment, we included ringing of the ears as a symptom in an attempt to determine whether respondents would answer yes to any symptom even if that symptom would not have been experienced as a result of chlorine exposure. Patients who reported that they experienced ringing of the ears had lower kappa coefficients for every symptom except dizziness than patients who did not report ringing of the ears. The average weighted kappa coefficient for all symptoms was 0.14 for those reporting ringing of the ears and 0.36 for those not reporting this symptom. Of the 85 discordant answers given by persons reporting ringing of the ears, $77(91 \%)$ answered no on the rapid assessment and yes on the follow-up assessment when the person was asked whether he/she experienced a symptom after the chlorine exposure; only 8 (9\%) answered yes on the rapid assessment and no on the follow-up. The 147 discordant answers given by persons not reporting ringing of the ears were more evenly distributed, with 83 $(56 \%)$ no/yes and 64 (44\%) yes/no.

Posttraumatic Stress Disorder Checklist for a Specific Event

Scores on the PCL-S ranged from 17 to 83 . Forty-four of $92(48 \%)$ respondents screened positive for PTSD. An analysis for risk factors associated with screening positive for PTSD is displayed in Table 5. Screening positive for PTSD was associated with having a severe medical outcome, still experiencing symptoms at the time of the follow-up, being under a physician's care, and taking medicine for symptoms related to chlorine exposure. Having a moderate-to-extreme exposure to chlorine was not associated with PTSD, nor was patient sex, race/ ethnicity $(p=0.26)$, or patient category [where the person was exposed to the chlorine (e.g., working at the mill or being a Graniteville resident), $p=0.38]$. Answering yes to experiencing ringing of the ears after chlorine exposure was 
Table 4 Kappa coefficient describing agreement between report of acute symptoms experienced in rapid assessment and follow-up assessment, stratified by those who reported that they experienced ringing of the ears after chlorine exposure and those who reported that they did not

\begin{tabular}{|c|c|c|c|}
\hline \multirow[t]{2}{*}{ Symptom } & \multicolumn{3}{|c|}{$\mathrm{Kappa}^{\mathrm{a}}$} \\
\hline & All & Reported ear ringing $^{\mathrm{b}}$ & Reported no ear ringing \\
\hline Chest pain & 0.56 & 0.44 & 0.65 \\
\hline Short of breath & 0.49 & 0.17 & 0.60 \\
\hline Eye burning & 0.44 & 0.29 & 0.48 \\
\hline Phlegm & 0.32 & 0.03 & 0.44 \\
\hline Choking & 0.32 & 0.31 & 0.38 \\
\hline Dizziness & 0.31 & 0.23 & 0.19 \\
\hline Headache & 0.30 & 0.16 & 0.38 \\
\hline Nausea & 0.25 & 0.01 & 0.35 \\
\hline Coughing & 0.13 & -0.07 & 0.25 \\
\hline Vomiting & 0.11 & -0.06 & 0.20 \\
\hline Nose burning & 0.06 & 0.03 & 0.08 \\
\hline Total $^{\mathrm{c}}$ & 0.30 & 0.14 & 0.36 \\
\hline
\end{tabular}

${ }^{a}$ Kappa coefficient values of $0.4-0.75$ indicate moderate agreement and values $<0.4$ indicate poor agreement

${ }^{\mathrm{b}}$ Ringing of the ears has not been identified in the literature as a symptom of chlorine exposure and was not reported by any of the participants during the rapid assessment. It was included in the follow-up assessment to try to determine if respondents would answer yes to any symptom listed on the questionnaire

${ }^{\mathrm{c}}$ Weighted average

associated with screening positive for PTSD [relative risk $(\mathrm{RR})=3.4 ; 95 \%$ confidence interval $(\mathrm{CI})=2.1-5.5]$. Of the 26 persons reporting ringing of the ears, $23(88 \%)$ screened positive for PTSD, whereas only $13(26 \%)$ of the 50 persons not reporting ear ringing screened positive.

\section{Discussion}

This follow-up assessment was undertaken primarily to determine the status of the persons exposed to chlorine, to provide assistance to respondents if needed, and to help in planning the Community Health Survey in the area. It also afforded the opportunity to compare the symptoms each person reported during the rapid assessment in the weeks after the exposure to the symptoms he or she recalled experiencing 5 months later. We found poor agreement between report of acute symptoms experienced on the rapid assessment and report of acute symptoms experienced on the follow-up assessment. The answer given closest to the time of the event was more likely to be accurate when answers were discordant since the potential existed for recall bias during the follow-up assessment. Because this assessment was performed to quickly gather data for public health action, reviewing medical chart to confirm self-report of symptoms and medical treatment was beyond its scope.

When performing long-term follow-up of persons exposed to a chemical release, a delay between the time of the exposure and the organization of the study can occur. Data regarding symptoms experienced might not be collected for months after the incident. The accurate recall of symptoms decreases with time, and victims might over- or underreport symptoms when
Table 5 Risk factors associated with screening positive for posttraumatic stress disorder

\footnotetext{
${ }^{a}$ Ringing of the ears, a symptom not known to be associated with chlorine exposure and not reported by any participants during the rapid assessment, was included as an indicator of overreporting
}

\begin{tabular}{lll}
\hline Risk factor & RR & $95 \%$ CI \\
\hline Experiencing symptoms at time of follow-up assessment & 9.1 & $1.3-61.2$ \\
Difficulty breathing since chlorine exposure limits walking & 4.9 & $2.1-11.2$ \\
Being under a physician's care for chlorine-related symptoms & 4.3 & $2.2-8.1$ \\
Ringing of the ears ${ }^{\mathrm{a}}$ & 3.4 & $2.1-5.5$ \\
Taking medication for problems related to the chlorine & 2.9 & $1.7-5.0$ \\
Effects of chlorine kept out of work (for any period) & 2.4 & $1.3-4.3$ \\
Attending community meetings & 1.8 & $1.2-2.7$ \\
Severe medical outcome & 1.7 & $1.1-2.6$ \\
Moderate-to-extreme chlorine exposure & 1.2 & $0.8-1.8$ \\
Sex (male versus female) & 1.1 & $0.7-1.7$ \\
\hline
\end{tabular}


the incident is no longer forefront in their minds. If data regarding acute symptoms is not collected for months after the exposure, investigators should consider information sources less prone to recall bias (e.g., medical records).

Including a symptom not known to be associated with the exposure on a questionnaire (e.g., our question inquiring about ringing of the ears) can help determine if overreporting occurs. Among symptoms reported by patients who reported ringing of the ears, discordant answers were heavily weighted toward reporting symptoms experienced on the follow-up that had not been reported on the rapid assessment. Among those not reporting ear ringing, the incongruous answers were nearly evenly distributed. This indicates that during the follow-up assessment, those reporting ear ringing might have been overreporting symptoms experienced during the incident. However, ringing of the ears might not have been a good symptom to use for a chemical with a respiratory exposure. During the follow-up assessment, one respondent listed chronic sinus problems since the chlorine exposure and another noted that his ears stay "stopped up." If chlorine exposure led to sinus infections that spread to the ears, ear ringing might be a consequence. Because we did not ask which medications respondents were taking, we were unable to explore if tinnitus was a side effect of drugs taken to treat symptoms of the chlorine exposure.

Although screening positive with the PCL-S could be indicative of PTSD, the high proportion of respondents who had a positive result on the screen should be interpreted with caution. First, the group who participated in the follow-up assessment was sicker, on average, than the group who had sought medical care after the derailment, as measured by our severe medical outcome categorization. Persons experiencing symptoms indicative of PTSD might have also been more likely to participate in the follow-up assessment. In addition, the poor kappa coefficient between the rapid and follow-up assessments and the likelihood that physical symptoms were being overreported suggest that psychological symptoms were also overreported. Despite the possibility of overreporting, we can conclude that chronic mental health symptoms were being experienced in Graniteville the summer after the train derailment, especially among those who had experienced the most severe medical outcome after the chlorine exposure and among those who were continuing to live with the physical effects of the chlorine. Consequently, a social worker was included on staff for the planned DHEC Community Health Survey.

\section{Limitations}

The main limitation of the rapid assessment was our difficulty locating persons treated for chlorine inhalation. This resulted in a higher proportion of persons experiencing a severe medical outcome being interviewed than those with a less severe outcome because they were still hospitalized 2 days after the chlorine spill and readily accessible when the rapid assessment commenced. This limitation might have been compounded by the low participation rate of the follow-up assessment. Persons still experiencing either physical or mental symptoms probably participated at a higher rate than those who had completely recovered, leading to selection bias based on persistent symptoms. Therefore, the results of the follow-up assessment cannot be assumed to be representative of all persons exposed to chlorine in the Graniteville derailment.

\section{Conclusion}

Self-report of symptoms experienced after a chemical exposure should be obtained as soon as possible if a longterm study is planned because recall might diminish within months. Symptoms unrelated to the exposure should be included in the symptom list to aid in the identification of overreporting. Five months after a substantial chlorine spill in Graniteville, South Carolina, multiple persons continued to experience physical symptoms and require medical care. In addition, persons were experiencing psychological symptoms indicative of PTSD.

Acknowledgments The authors acknowledge the support and assistance of the following persons: Jill Ferdinands, Marge Heim, Pat McMahan, Phillipine Outing, Veleta Rudnick, and Rosa Wesley. The findings and conclusions in this report are those of the author(s) and do not necessarily represent the views of the Centers for Disease Control and Prevention.

\section{References}

1. Wenck MA, van Sickle D, Drociuk D, Belflower A, Youngblood C, Whisnant MD et al (2007) Rapid assessment of exposure to chlorine released from a train derailment and resulting health impact. Public Health Rep 122:784-792

2. Van Sickle D, Wenck MA, Belflower A, Drociuk D, Ferdinands J (2007) Panel classification of self-reported exposure histories: a useful exposure index after a mass-casualty event. Public Health Rep 122:766-783

3. National Center for PTSD. http://www.ptsd.va.gov/professional/ pages/assessments/ptsd-checklist.asp, accessed 19 Nov 2010

4. Weathers F, Litz B, Herman D, Huska J, Keane T (1993) The PTSD checklist (PCL): reliability, validity, and diagnostic utility. Paper presented at the Annual Convention of the International Society for Traumatic Stress Studies, San Antonio, TX

5. SAS Institute, Inc. (2002-2003) SAS $^{\circledR}$ : version 9.1. SAS Institute, Inc., Cary, NC

6. Centers for Disease Control and Prevention and World Health Organization (2001) Epi Info 6.04d. CDC/WHO, Atlanta, GA/ Geneva, Switzerland

7. Landis JR, Koch GG (1977) The measurement of observer agreement for categorical data. Biometrics 33:159-174 